\title{
SEROPREVALENCIA DE LEPTOSPIRA SPP. EN CANINOS DE LA CIUDAD DE CÓRDOBA, ARGENTINA.
}

M. del Rosario Rollán, María G. Irrazabal, ${ }^{2}$ Exequiel Scialfa, ${ }^{3}$ Germán Zurbriggen, ${ }^{4}$ Diego Graiff, ${ }^{5}$ Federico J. Giraudo, ${ }^{6}$ Susana E. Ruiz. ${ }^{7}$
'Mag. en Educación. Bioquímica. Farmacéutica. Investigadora. Facultad de Ciencias Químicas. Universidad Católica de Córdoba

${ }^{2}$ Médico Veterinario. Investigadora. Facultad de Ciencias Agropecuarias. Universidad Católica de Córdoba

${ }^{3}$ Doctor en Ciencia Animal.

Médico Veterinario. Investigador. Departamento de Zoonosis Rurales. Azul. Provincia de Buenos Aires ${ }^{4}$ Médico Veterinario. Investigador. Facultad de Ciencias Agropecuarias. Universidad Católica de Córdoba

${ }^{5}$ Mag. en Ciencias Veterinarias. Médico Veterinario. Investigador.

Facultad de Ciencias

Agropecuarias. Universidad Católica de Córdoba

${ }^{6}$ Mag. en Atención

Farmacéutica. Farmacéutico. Investigador. Facultad de Ciencias Químicas.

Universidad Católica de Córdoba

${ }^{7}$ Esp. en Bacteriología

Bioquímica. Investigadora.

Facultad de Ciencias

Químicas. Universidad Católica de Córdoba

Trabajo recibido: 23 de agosto 2018.

Aprobado: 18 de octubre2018.
SEROPREVALENCE OF LEPTOSPIRA SPP. IN DOGS, IN THE CITY OF CORDOBA, ARGENTINA.

\section{SEROPREVALÊNCIA DE LEPTOSPIRA SPP. EM CÃESDACIDADEDECÓRDOBA,ARGENTINA.}

\section{Resumen}

La leptospirosis, es una enfermedad transmisible, causada por una bacteria, Leptospira spp., considerada como la zoonosis de mayor difusión mundial. Se realizó un estudio para conocer la presencia de anticuerpos anti-Leptospira, en perros de compañía, de la ciudad de Córdoba, Argentina. Las muestras de sangre se obtuvieron a través de un muestreo de conveniencia no probabilístico. Se analizaron 102 muestras mediante la técnica de aglutinación microscópica (MAT), implementada con 10 serotipos. La prevalencia muestral de anticuerpos anti-Leptospira fue del $82.35 \%$. La mayor frecuencia fue para los serovares Hardjo, Castellonis y Canicola. La mayoría de las muestras fueron reactivas a un solo serotipo. Los resultados cobran relevancia debido a la falta de datos epidemiológicos en la zona, poniendo en evidencia la presencia de la bacteria en la población canina y la necesidad de establecer medidas de control y prevención en la región para esta zoonosis, tan importante en salud pública.

Palabras Clave: Leptospirosis, Zoonosis, Epidemiología.

\section{Abstract}

Leptospirosis is a communicative disease caused by a bacterium called Leptospira spp, considered the zoonosis of greatest world wide distribution. A study was carried out to find out about the presence of antibodies anti-Leptospira in companion dogs in the city of Cordoba, Argentina. Blood samples were obtained through convenience non probability sampling. Microscopic agglutination test 
(MAT) was used to analyze 102 samples, implemented with 10 serotypes. The sampling prevalence of antibodies anti-Leptospira was $82.35 \%$. The highest frequency corresponded to serovars Hardjo, Castellonis and Canicola.Most sample were reactive to only one serovar. Results are relevant due to the lack of epidemiological information in the area, showing evidence of bacteria in the population of dogs and the need to set up control and prevention measures for this zoonosis in the region, so important in public health.

Key words: Leptospirosis, Zoonosis, Epidemiology.

\section{Resumo}

A leptospirose, é uma doença transmissível, causada por uma bactéria, Leptospira spp., Considerada a zoonose de maior difusão mundial. Um estudo foi realizado para determinar a presença de anticorpos anti-Leptospira, em cães, mascotes de estimação, da cidade de Córdoba, Argentina. Amostras de sangue foram obtidas por amostragem de conveniência não probabilística. Foram analisadas 102 amostras utilizando a técnica de aglutinação microscópica (MAT), implementada com 10 sorotipos. A prevalência da amostra de anticorpos anti-Leptospira foi de $82,35 \%$. A maior frequência foi para os sorovares Hardjo, Castellonis e Canicola. A maioria das amostras foi reativa a um único sorotipo. Os resultados são relevantes devido à falta de dados epidemiológicos na área, evidenciando a presença da bactéria na população canina e a necessidade de estabelecer medidas de controle e prevenção na região para essa zoonose, tão importante na saúde pública.

Palavras chave: Leptospirose, Zoonose, Epidemiologia.

\section{Introducción}

La Leptospirosis es una enfermedad transmisible, considerada por la Organización Mundial de la Salud (WHO) y la Oficina Internacional de las Epizootias (OIE) como la zoonosis de mayor difusión en el mundo (1).

El ser humano es un huésped accidental y puede presentar cuadros clínicos variados, que van desde formas leves, hasta la muerte (2); si bien es considerada de baja mortalidad, pero de alta morbilidad (3).

Su agente etiológico es una bacteria, móvil, que pertenece al género Leptospira, de 0,1 micras de espesor por 7 a 14 micras de longitud, con extremos en forma de gancho.

El género Leptospira incluye, hasta el momento, según la clasificación filogenética, veintiuna especies diferentes organizadas en tres grandes subgrupos, aisladas de diversos ambientes. Dentro de las cuales, nueve son especies patógenas capaces de infectar y causar enfermedad en humanos y animales (Leptospira interrogans, Leptospira kirschneri, Leptospira borgpetersenii , Leptospira santarosai, Leptospira noguchii, Leptospira weilii, Leptospira alexanderi, Leptospira kmetyi y Leptospira alstonii); cinco son especies intermedias aisladas de humanos y animales y que pueden ser causales de manifestaciones clínicas leves (Leptospira inadai, Leptospira broomii, Leptospira fainei, Leptospira wolffii y Leptospira licerasiae); y siete especies son saprófitas, ambientales y no causan enfermedades en el hombre ni en los animales (Leptospira biflexa, Leptospira wolbachii, Leptospira meyeri, Leptospira vanthielii, Leptospira terpstrae, Leptospira idonii y Leptospira yanagawae) $(4,5)$.

Esta zoonosis, presenta una amplia variedad de reservorios, entre los que se encuentran muchas especies de mamíferos (6). Los roedores se consideran el principal reservorio silvestre, pero el perro actúa como un potencial diseminador, ya que mantiene una estrecha relación con el hombre, y al mismo tiempo con otros animales domésticos, y es capaz de ser portador asintomático de la misma (7).

A pesar de la gran importancia epidemiológica, la Leptospirosis canina, frecuentemente es 
subdiagnosticada, debido a que su principal forma de presentación es subclínica y cuando da sintomatología presenta una gran variedad de manifestaciones clínicas, lo cual dificulta su diagnóstico $(8,9)$.

La vacunación de perros y otros animales previene la enfermedad, pero no previene la infección o la trasmisión, siendo la vacuna, específica solamente para las serovariedades presentes en su formulación (10).

Los distintos serovares tienen predilección por algunas especies animales, por ejemplo: L. Canicola asociada al perro, L. Hardjo para bovinos, L. Pomona a bovinos y cerdos, L. Bratislava a cerdos y L. Icterohaemorrhagiae a roedores y perros, siendo ésta última la que produce con mayor frecuencia infección grave en humanos (11).

Las leptospiras se pueden transmitir directa o indirectamente, entre animales, o de los animales a las personas. La forma directa ocurre al entrar en contacto con sangre, tejidos u órganos de animales infectados, mientras que en la forma indirecta, que es la más frecuente, el agua juega un papel primordial, ya que las leptospiras provenientes de terrenos o agua contaminada con orina de animales enfermos, ingresan al organismo a través de la mucosa oral, conjuntival, nasal o genital; como también, a través de la piel con laceraciones o reblandecida por la humedad. La tasa de transmisión es muy elevada, ya que sólo diez microorganismos son necesarios para causar la patología (12).

Esta enfermedad constituye un importante problema emergente en la Salud Pública. El cambio climático que vivimos a nivel mundial y el advenimiento de las inundaciones, favorece la presencia de estas bacterias en el ambiente, lo cual podría aumentar la incidencia de casos de leptospirosis si no se toman los recaudos sanitarios con la debida anticipación $(13,14,15)$.

Poco se conoce de la realidad epidemiológica de esta enfermedad en la Provincia de Córdoba y menos aún en áreas peri-urbanas de la ciudad. En mayo del 2018, en el Boletín Integrado de Vigilancia de la Secretaría de Promoción y Programas Sanitarios del Ministerio de Salud de la Nación Argentina, la Provincia de Córdoba notificó durante los años 2017 y 2018, 30 y 28 casos respectivamente de Leptospirosis, confirmando solo 2 casos en el año 2017, lo que muestra el escaso conocimiento del perfil epidemiológico de la enfermedad en la zona.

El objetivo del presente trabajo fue conocer la presencia de anticuerpos anti-Leptospira en perros residentes en la zona sudoeste, área urbana periférica de la ciudad de Córdoba, (Córdoba, Argentina), de marzo a septiembre de 2016, lo cual permitiría generar información oportuna, válida y confiable para orientar medidas de prevención y diseñar políticas públicas de control a nivel local.

\section{Desarrollo}

A través de un muestreo por conveniencia (16), empleando un estudio descriptivo, exploratorio y transversal, se estudió una muestra de 102 caninos durante los meses de marzo a septiembre de 2016, residentes en la zona urbana periférica del sudoeste de la ciudad de Córdoba. Se excluyeron animales agresivos y animales menores de tres meses. A cada persona responsable o propietario de la mascota, se le solicitó el consentimiento y se le realizó una encuesta, en la que se consignaron datos como: especie, sexo, edad, tamaño, castración, antecedentes de vacunación y síntomas de enfermedades, entre otros. El estudio contó con la aprobación del Centro de Bioética. Ninguno de los perros muestreados había sido vacunado contra leptospirosis.

Ante la imposibilidad de definir a priori el tamaño muestral, debido al tipo de muestreo por conveniencia y al prerrequisito de contar con el consentimiento de los dueños, se tomó la estrategia de recoger la mayor cantidad posible de observaciones y definir como variable dependiente el error muestral inherente al estudio. Siguiendo a Berenson, Levine y Krehbiel (17), en función a la siguiente expresión: 


$$
e=\sqrt{\frac{P(1-P) z_{\alpha / 2}{ }^{2}}{n}}=\sqrt{\frac{0.530 .471 .96^{2}}{102}}=0.0967
$$

En donde $\mathrm{n}$ es el tamaño muestral obtenido, $\mathrm{P}$ es la tasa de prevalencia observada en estudios previos* fijada en 0,53 y trabajando con un nivel de confianza igual al 95\%, el error muestral e inherente al presente estudio resultó igual al 9.7\%.

La muestra estuvo compuesta por 41 perros que fueron llevados a una jornada de vacunación contra la rabia, realizada en una Plaza de barrio Cabildo, ubicado en la zona sudoeste de la ciudad de Córdoba, Argentina, y que se encuentra aproximadamente a 3300 metros de uno de los basurales de la Ciudad (Enterramiento Sanitario Piedras Blancas $31^{\circ} 31^{\prime} 02.7^{\prime}$ 'S 6413'54.7'W) y 61 muestras fueron de animales, pertenecientes a vecinos del barrio, que consultaron por diversas razones a dos veterinarias de la zona. (Figura 1)

Figura 1: Imagen satelital de la ciudad de Córdoba, localización de los puntos de toma de muestra y del Enterramiento Sanitario Piedras Blancas.

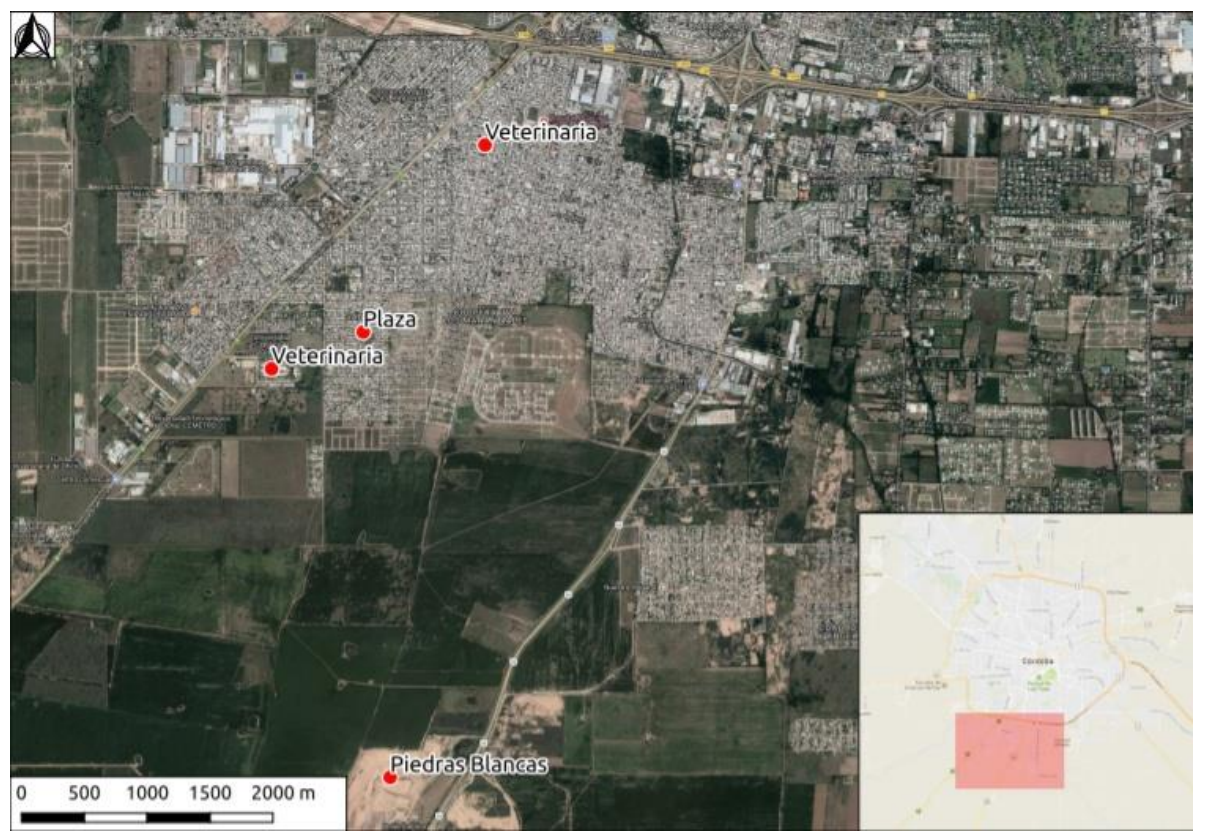

Se verificó el consentimiento firmado por los propietarios de las mascotas, a los fines de evitar la duplicación de observaciones en la muestra.

Se obtuvieron muestras de sangre por punción de vena cefálica antebraquial o yugular, obteniendo $5 \mathrm{~mL}$ en tubos estériles, rotulados, sin anticoagulante. Las muestras fueron transportadas refrigeradas hasta el laboratorio, donde se centrifugaron a $4000 \mathrm{rpm}$ durante 5 minutos. El suero fue almacenado en tubos Eppendorf y congelados a $-70^{\circ} \mathrm{C}$.

Para su procesamiento, las muestras, fueron trasladadas en forma congelada manteniendo la cadena de frío (hielo seco), en triple envase rotulado, a un Laboratorio de Referencia de la Provincia de Buenos Aires, donde se realizó el diagnóstico mediante la técnica de aglutinación microscópica. Se utilizaron cepas de referencia de L. interrogans serovares Canicola, Copenhageni, Pomona, Pyrogenes, Wolffi, Hebdomadis y Hardjo; L. borgpeterseni serovares Tarassovi y Castellonis y L. kirschneri serovar Grippotyphosa, vivas desar-

*La tasa de prevalencia según diferentes autores fue de: Scialfa E y col: 65\%; Scialfa E: 91\%; Rubel D y col: 57\%; Tealdo MS y col: 33\%; Scialfa E y col: $80.4 \%$; Cramer AN y col: $18.8 \%$; Gualtieri C y col: $64 \%$; Tuemmers C y col: $21.3 \%$. La tasa de prevalencia promedio en estos estudios es igual a 0.53 . 
rolladas a $28-30^{\circ} \mathrm{C}$ en medio EMJH y con no más de 15 días de crecimiento. Se consideró un suero positivo cuando se observó aglutinación del $50 \%$, en una dilución $\geq 1: 100$ para uno o más serovares (18).

En base a los datos muéstrales relevados, se procedió a elaborar una serie de estadísticas descriptivas, en Microsoft Excel versión 2013 para Windows TM, que fueron utilizadas para inferir los valores poblacionales de la prevalencia de Leptospira spp. mediante la estimación de un intervalo de confianza, con un nivel de confianza igual al 95\%.

\section{Resultados}

De un total de 102 muestras de suero, 84 fueron reactivas $(82.35 \%), 17$ no reactivas (16.67\%) y 1 muestra fue descartada por contaminación $(0.98 \%)$. En base a la distribución de probabilidad de la proporción muestral, estos resultados permiten afirmar con un nivel de confianza igual al $95 \%$, que la tasa de prevalencia en la población estará comprendida por el intervalo $74.9 \%-89.7 \%$.

La mayor frecuencia de hallazgo en las muestras reactivas fue para los serovares Hardjo (89.28\%), seguido por Castellonis (44.05\%) y Canicola (35.71\%). Ninguna muestra fue reactiva para los serovares Pyrogenes, Pomona y Wolffi (Tabla 1).

Tabla $\mathbf{N}^{\mathbf{0}}$ 1: Frecuencia de serovares en muestras reactivas

\begin{tabular}{lcc}
\hline SEROVARES & $\mathrm{N}^{\mathrm{o}}$ & $\%$ \\
\hline Castellonis & 37 & 44.05 \\
Canícola & 30 & 35.71 \\
Grippothyosa & 18 & 21.43 \\
Hardjo & 75 & 89.28 \\
Hebdomadis & 21 & 25 \\
Copenhageni & 7 & 8.33 \\
Tarassovi & 2 & 2.38 \\
Total & 84 & 100 \\
\hline
\end{tabular}

De las 84 muestras reactivas, el $44.05 \%$ lo fue para un serovar, con predominio de Hardjo; el $20.24 \%$ a dos y cuatro o más serovares y por último, el $15.47 \%$, reaccionó a tres serovares (Tabla 2, Tabla 3 y Tabla 4).

Tabla $\mathbf{N}^{0}$ 2: Frecuencia de serovares en muestras reactivas a 1 serovar

\begin{tabular}{lcc}
\hline SEROVARES & $\mathrm{N}^{\mathrm{o}}$ & $\%$ \\
\hline Castellonis & 4 & 10.82 \\
Hardjo & 31 & 83.78 \\
Grippothyosa & 1 & 2.7 \\
Copenhageni & 1 & 2.7 \\
Total & 37 & 100 \\
\hline
\end{tabular}

Tabla $\mathbf{N}^{\mathbf{0}}$ 3: Frecuencia de serovares en muestras reactivas a 2 serovares

\begin{tabular}{lcc}
\hline SEROVARES & $\mathrm{N}^{\mathrm{o}}$ & $\%$ \\
\hline Castellonis/Canicola & 2 & 11.76 \\
Hardjo/ Canícola & 3 & 17.65 \\
Castellonis/Hardjo & 4 & 23.53 \\
Hardjo/ Hebdomadis & 7 & 41.18 \\
Copenhageni & 1 & 5.88 \\
Total & 17 & 100 \\
\hline
\end{tabular}


Tabla $\mathbf{N}^{\circ}$ 4: Frecuencia de serovares en muestras reactivas a 4 o más serovares

\begin{tabular}{lcc}
\hline SEROVARES & TOTALES & $\%$ \\
\hline Castellonis/Canicola/Hardjo/Hebdomanis/Grippotyphosa & 9 & 52.95 \\
Castellonis/Canícola/Grippotyphosa/Hardjo & 4 & 23.53 \\
Castellonis/Hardjo/Grippotyphosa/Copenhageni & 1 & 5.88 \\
Castellonis/Canicola/Hardjo/Hebdomadis & 1 & 5.88 \\
Castellonis/Canicola/Hardjo/Grippotyphosa/Copenhageni & 1 & 5,88 \\
Castellonis/Canicola/Hardjo/Tarassovi/Grippotyphosa & 1 & 5.88 \\
Total & 17 & 100 \\
\hline
\end{tabular}

Los patrones de coaglutinación más frecuentes fueron a Castellonis-CanicolaGryppotyphosa-Hardjo-Hebdomadis.

\section{Conclusiones}

Los hallazgos obtenidos en este estudio, mostraron la existencia de circulación de leptospiras en la zona, obteniendo para la prueba serológica un $82.35 \%$ de positividad en la muestra de caninos estudiada, valor que permite inferir una tasa de prevalencia en la población canina entre $74.9 \%$ y $89.7 \%$. Este resultado se asemeja a lo informado en caninos de áreas peri-urbanas del interior de la provincia de Buenos Aires, donde los sueros fueron reactivos entre el $65 \%$ y $91 \%$ para Leptospiras spp $(19,20)$.

En el Gran Buenos Aires, el 57\% de los sueros de caninos han sido positivos, siendo los serogrupos Canicola y Pyrogenes los detectados con mayor frecuencia (21). En la misma provincia, en perros atendidos por distintos motivos en el Instituto de Zoonosis Luis Pasteur, se encontró una seroprevalencia del 33\% y como dato inédito, el 59\% de los sueros reactivos lo fueron al serovar Cynopteri, seguidos por Castellonis, Canicola y Pyrogenes (22). En un refugio canino del partido de Dorrego, Buenos Aires, se encontró una prevalencia para esta bacteria del $80.4 \%$ con sueros reactivos a serovares Castellonis, Gryppotyphosa, Canicola, Hebdomadis, Hardjo, Copenhageni y Tarassovi (23).

En Esperanza, provincia de Santa Fe, luego de una inundación se obtuvieron muestras de los perros de la zona afectada y se halló un $18.8 \%$ de sueros reactivos, siendo el serovar Canicola el de mayor frecuencia (24). En el año 2012 en la ciudad de Rosario, se encontró un $64 \%$ de perros reactivos, cuyos serovares prevalentes fueron Castellonis, Icterohaemorrhagiae, Canicola y Pyrogenes (25). Datos similares se encontraron en la misma ciudad con 22 animales positivos de 156 estudiados, siendo Castellonis el serovar más frecuente, al cual le siguieron Canicola, Pyrogenes, Pomona, Icterohaemorrhagiae (26).

Otros autores han hallado seroprevalencias del $21.3 \%$ en perros vagabundos de la ciudad de Temuco, Chile (27).

Un hallazgo en este estudio, que difiere a lo encontrado por los investigadores anteriormente mencionados, es la alta frecuencia del serovar Hardjo, seguido por Castellonis y Canicola. Este serovar se relaciona principalmente a bovinos, sin embargo, en caninos provenientes de un albergue para animales callejeros del estado Aragua, Venezuela, se lo encontró como serovar más frecuente junto a Canicola e Icterohaemorrhagiae (28), como también en una investigación realizada en caninos callejeros en la ciudad de Maracay, estado Aragua, Venezuela, se observó una alta frecuencia de Hardjo en el medio urbano (29).

Por tratarse el área estudiada, de un zona peri-urbana de la capital provincial, cercana a un enterramiento de basura, este resultado podría estar relacionado con diversos factores, por ejemplo, el mayor contacto de los animales con reservorios silvestres; la presencia de algún matadero clandestino que favorezca el contacto con el agua contaminada por la orina del ganado y/o cerdos o por su sangre y/o tejidos infectados desechados, o a la existencia de carreros que juegan un rol importante con la tracción a sangre de los caballos 
y la introducción de los mismos a la ciudad, debido a que el serovar Hardjo también se puede aislar de esta especie animal (30).

En conclusión, la reactividad serológica que presentaron los animales estudiados, pone en evidencia la presencia de esta bacteria en la zona y señala a la población canina como un importante factor de riesgo para la salud pública, ya que se ha demostrado que el contacto de los perros domésticos con un entorno al aire libre, zanjas cercanas y otros perros o especies, entre otras cosas, es favorable para la infección y propagación del microorganismo.

Los resultados obtenidos en las distintas investigaciones, permiten concluir que tanto las tasas de infección como la reacción para los diferentes serovares son dependientes del área de residencia de los animales.

Debido a la importancia de los datos obtenidos, se intervino en la zona desde un Programa de Responsabilidad Social Universitaria en la concientización y en las distintas medidas preventivas que aportan a la salud colectiva de la población, informando a los propietarios de las mascotas y a la población en general, sobre los riesgos de convivencia diaria con animales, si estos no son cuidados en forma responsable, las medidas de manejo e higiene y los mínimos controles médicos veterinarios necesarios para prevenir enfermedades zoonóticas, como también en la necesidad de mantener una buena higiene ambiental, control de roedores y adecuado manejo de la basura.

Se sugiere la realización de investigaciones con el objeto de aislar y tipificar las cepas endémicas, esto permitiría incluirlas en los ceparios de diagnóstico, conocer la epidemiología en la zona y aportar en la formulación de vacunas, como también, establecer cuáles son los factores de riesgo para los caninos en la zona y cuales las especies animales que son fuente de infección para orientar medidas de control del reservorio local.

\section{Bibliografía}

1. Silva J, Rivero M, Scialfa EK; Epidemiología de la leptospirosis humana de un área rururbana del partido de Tandil. Documento de conferencia. IV Congreso Internacional Científico y Tecnológico-CONCYT. Buenos Aires, Argentina. Septiembre 2017. Disponible en: https://digital.cic.gba.gob.ar/bitstream/ handle/11746/6716/11746_6716.pdf?sequence=1\&isAllowed=y Acceso el 31 de julio de 2018.

2. Chávez A, Flores Somarriba B, Soto A, Sheleby-Elías J, Duttmann Ch, Jiménez E, et al. Detección de Leptospira spp. en animales y muestras ambientales de áreas peridomésticas en Nicaragua. Rev Panam Salud Pública. 2018; 42:e26. Disponible en: https://doi.org/10.26633/RPSP.2018.26 Acceso el 31 de julio de 2018.

3. Adler B, Lo M, Seemann T, Murray G; Pathogenesis of leptospirosis: The influence of genomics. Vet Microbiol 2011; 153: 73-81.

4. Bourhy, P., Collet, L., Brisse, S., y Picardeau, M. Leptospira mayottensis sp. nov., una especie patógena del género Leptospira aislada de humanos. Revista Internacional de Microbiología Sistemática y Evolutiva. 2014; 64 (Pt 12), 40614067. Disponible en: http://doi.org/10.1099/ijs.0.066597-0 Acceso 31 de julio de 2018.

5. OPS OMS; Leptospirosis (información detallada). 2017 Disponible en: https:// www.paho.org/hq/index.php?option $=$ com_content $\&$ view $=$ article $\&$ id $=7377 \%$ 3A2012-leptospirosis-informacion-detallada\&catid=4784\%3 Aleptospirosiscontents\&Itemid=39617\&lang=es Acceso el 31 de julio de 2018.

6. Ochoa JE, Sanchez A, Ruiz I; Epidemiologia de la leptospirosis en una zona andina de producción pecuaria. Rev Panam Salud Pública. 2000; 7(5) Disponible en:http://www.scielosp.org/scielo.php?script=sci_arttext\&pid=S102049892000000500006\&lng=pt\&nrm=iso Acceso el 31 de julio de 2018. 
7. Sant>ana R, Vieira A, Grapiglia J, Lilenbaum W; High number of asymptomatic dogs as leptospiral carriers in an endemic area indicates a serious public health concern. Epidemiology and Infection. 2017; 145(9), 1852-1854.

8. Levett P; Leptospirosis: A forgotten zoonosis? Clin Applied Immunol Rev. 2004; 4: 435-48.

9. Luna A M A, Moles C L P, Gavaldón R D, Nava VC, Salazar GF; La Leptospirosis canina y su problemática en México. Rev Salud Anim. 2008; 30(1):01-11. Disponibleen:http://scielo.sld.cu/scielo.php?script=sci_arttext\&pid=S0253570X2008000100001\&lng=es Acceso el 31 de julio de 2018.

10. Romero-Vivas C M, Falconar A K; Leptospira spp. y leptospirosis humana. Salud, Barranquilla. 2016; 32(1):123-143. Disponible en: http://dx.doi.org/10.14482/ sun.32.1.8479 Acceso el 21 de junio de 2018.

11. Tabío Y, Palmero Y, Cruz E; Human Leptospirosis and risk factors Revista Científica Infociencia. 2010; 14 (3): 1-11.

12. Dabanch J; Zoonosis. Rev Chilena Infectol. 2003; 20 (1): 47-51 Disponible en:https://scielo.conicyt.cl/scielo.php?script=sci_arttext\&pid=S0716$10182003020100008 \& \operatorname{lng}=$ es Acceso 31 de julio de 2018.

13. Hartskeerl R, Collares-Pereira M, Ellis W; Emergence, control and re-emerging leptospirosis: dynamics of infection in the changing world. Clin Microbiol Infect. 2011; 17 (4): 494-501. Disponible en: https://www.clinicalmicrobiologyandinfecti on.com/article/S1198-743X(14)63264-X/pdf Acceso 31 de julio de 2018.

14. OPS OMS; Información general: Leptospirosis. 2017 Disponible en: https:// www.paho.org/hq/index.php?option $=$ com_content\&view $=$ article\&id $=7821$ \%3A2012-informacion-general-leptospirosis\&catid=4784\%3Aleptospirosiscontents\&Itemid $=0$ \&lang $=$ es

Acceso el 31 de julio de 2018.

15. Coelho M, Massad E; The impact of climate on Leptospirosis in São Paulo, Brazil. Int J Biometeorol. 2012; 56 (2): 233-41.

16. Martin SW, Meek AH, Willeberg P; Epidemiología veterinaria: Principios y métodos. Zaragoza (España): Ed. Acribia S.A., 1997. 398 p.

17. Berenson, Levine y Krehbiel, "Estadística para Administración”, Pearson Educación, 2a Ed., México, 2001.

18. Jacob P, Schmeling MF, Chiani YT, Landolt NY, Scialfa E, Fusco S et al; Evaluación de un panel reducido de cepas de leptospiras para el diagnóstico en humanos por microaglutinación (MAT). Salud pública Méx. 2015; 57(5):419-425. Disponible en:http://www.scielo.org.mx/scielo.php?script=sci_arttext\&pid=S0036$36342015000500013 \& \operatorname{lng}=$ es Acceso el 31 de julio de 2018.

19. Scialfa E, Gallicchio O, Grune S, Recavarren M, Rivero M, Quintana S, Aguirre P, San Antón D, Brihuega B; Actividades de vigilancia epidemiológica de la leptospirosis en el interior de la provincia de Buenos Aires. Rev Arg Zoonosis y Enf Infecc Emerg 2017; 12 (1):1-6.

20. Scialfa E; Los caninos: reservorios de leptospiras y posible fuente de infección para los seres humanos en el interior de la provincia de Buenos Aires. XVIII Simposio Internacional sobre enfermedades desatendidas. Buenos Aires, Argentina. 5 y 6 de octubre de 2017; pp. 38 Disponible en: https://www.mundosano.org/wp-content/ uploads/2017/10/libro_resumenes_2017.pdf Acceso el 31 de julio de 2018

21. Rubel D, Seijo A, Cernigoi B, Viale A y Wisnivesky-Colli C; Leptospira interrogans en una población canina del Gran Buenos Aires: variables asociadas con la seropositividad. Rev Panam Salud Pública. 1997; 2:102-5 Disponible en: https://www.scielosp.org/article/rpsp/1997.v2n2/102-106/ Acceso el 31 de julio de 2018.

22. Tealdo MS, Romero GN, Autrey CD, Samartino L; Serología positiva a Leptospira 
interrogans, serovar cynopteri en caninos de la Ciudad de Buenos Aires, Argentina. InVet. 2007; 9(1):59-65.

23. Scialfa E, Grune S, Brihuega B, Viscay R, San Anton D, Gallicchio $\mathrm{O}$; Relevamiento serológico de leptospirosis en caninos de un refugio canino del partido de Dorrego. XVII Simposio Internacional sobre enfermedades desatendidas. Buenos Aires, Argentina. 24 y 25 de agosto de 2015; pp. 74. Disponible en: https://docplayer.es/17117974Resumenes-de-presentaciones-24-y-25-de-agosto-de-2015-academianacional-de-medicina-caba.html Acceso el 31 de julio de 2018

24. Cramer AN, De Gennaro M, Miño K,Pepino S, Ruiz M, Schmeling F, Tomatis FM, Vanasco NB y Widenhorn N; Seroprevalencia de Leptospirosis en caninos expuestos a una inundación en Esperanza, Santa Fe, Argentina 2007. Revista FAVE - Ciencias Veterinarias. 2009; 8 (1):7-9. Disponible en: file:///C:/Users/Usuario/Downloads/1474Texto\%20del\%20art\%C3\%ADculo-3882-1-10-20140210.pdf Acceso el 31 de julio de 2018.

25. Gualtieri CAS,Carlín C,Peralta 1,Peirone C,Gattarello V,Marc L,Molteni H,Arestegui MB,François S; Evaluación clínica, bioquímica y hematológica de caninos seropositivos a distintos serovares de Leptospira interrogans. InVet. 2012; 14(2):131-139 Disponible en: http://www.fvet.uba.ar/fcvanterior/publicaciones/archivos/vol_14_ n2_2012/Vol-14N_2_2012_ARTICULO_1.pdf Acceso el 31 de julio de 2018 .

26. Seghesso Zabala A, Anthony Omezzolli LM, Poli Lovagnini G, Francois Barbagelata S; Seropositividad a Leptospira interrogans en perros de la ciudad de Rosario, Argentina. Rev Cubana MedTrop. 2013; 65(2):185-190. Disponible en: http://scielo.sld.cu/scielo. php?script $=$ sci_arttext\&pid $=$ S0375-07602013000200005\&lng=es Acceso el 25 de junio de 2018.

27. Tuemmers C, Lüders C, Rojas C, Serri M, Espinoza R y Castillo C; Prevalencia de leptospirosis en perros vagos capturados en la ciudad de Temuco, 2011. Rev Chilena Infectol. 2013; 30(3): 252-257 Disponible en: https://scielo.conicyt.cl/scielo.php?script=sci_arttext\&pid=S071610182013000300003\&lng=es Acceso el 31 de julio de 2018

28. Medina A, Zuleima, Guerra B, María, y Veliz, Noél; Estudio serológico de leptospirosis en caninos de un albergue en el estado Aragua. Revista de la Facultad de Ciencias Veterinarias. 2010; 51(2):093097. Disponible en: http://www.scielo.org.ve/scielo.php?script=sci arttext\&pid=S0258-65762010000200004\&lng=es\&tlng=es. Acceso el 31 de julio de 2018.

29. Medina Z, Guerra M; Seroprevalencia de Leptospira spp. en Caninos Callejeros de la Parroquia Madre María de San José, Municipio Girardot estado Aragua. Rev. Fac Cs. Vets.UCV. 2005; 46:1-8. Disponible en: http://www.scielo.org.ve/scielo.php?script=sci_arttext \&pid=S0258-65762005000100001 Acceso el 31 de julio de 2018.

30. Caselani K, Oliveira PR, Ferraudo AS, Lima-Ribeiro AMC, Girio RJS; Estudo soroepidemiológico de leptospirose em equinos utilizados para tração urbana. Revista do Instituto Adolfo Lutz. 2012; 71(3):582-587. Disponible en: http://periodicos.ses.sp.bvs.br/pdf/rial/v71n3/v71n3a20. pdf Acceso el 31de julio de 2018. 